\title{
Hot Gas and Physical Structure of 30 Dor
}

\author{
Q. Daniel Wang \\ Dept. of Physics \& Astronomy, Northwestern University, 2145 Sheridan \\ Road, Evanston, IL 60208-3112, USA
}

\begin{abstract}
Recent $R O S A T$ and $A S C A$ X-ray observations as well as $H S T$ images have provided new insights into the structure and evolution of the 30 Dor nebula. I review hot gas properties of the nebula and discuss related physical processes. The structure of the nebula can be understood as outflows of hot and HII gases from the parent giant molecular cloud of R136. The dynamic mixing between the two gas phases is likely a key mass loading process of the hot gas, providing a natural explanation of both temperature and density structure of the nebula.
\end{abstract}

\section{Introduction}

Hot gas in the 30 Dor nebula was first detected by the Einstein Observatory (Wang \& Helfand 1991; Chu \& Mac Low 1990). The nebula apparently consists of blisters of hot gas surrounded by $\mathrm{H} \alpha$-emitting filaments. Stellar winds and supernova explosions are presumably responsible for the heating of the gas. Recently, the nebula has been observed with instruments on board the ROSAT and $A S C A \mathrm{X}$-ray Observatories. X-ray images from some of these observations have been presented (Chu 1993; Wang 1995a; Wang \& Gotthelf 1998), but detailed analysis of the data is still ongoing. In this paper, I review some preliminary results on hot gas properties and discuss implications on various relevant physical processes.

\section{Hot Gas Properties}

Fig. 1 shows a state-of-art high resolution $\mathrm{X}$-ray image of the 30 Dor nebula (Wang 1999). In its core region, two point-like sources with $0.1-2.4 \mathrm{keV}$ luminosities of $\sim 10^{36} \mathrm{ergs} \mathrm{s}^{-1}$ stand out and are most likely Wolf-Rayet + black hole binary systems (Wang 1995a). The presence of such systems indicates that supernovae have occurred in the recent past of 30 Dor. The X-ray contribution from the central star cluster R136 is also detected (Fig. 1). The image shows no evidence for other point-like $\mathrm{X}$-ray sources which may be related to 30 Dor. The detection limit is $\sim 10^{35} \mathrm{ergs} \mathrm{s}^{-1}$. There is also no correlation between the X-ray intensity and the stellar distribution. The X-ray emission is thus predominantly diffuse in origin. A three color composite image of the nebula in $\mathrm{H} \alpha, \mathrm{UV}$, and $\mathrm{X}$ ray can be found at http://www.astro.nwu.edu/astro/wqd/images/30d.htm. Wang \& Gotthelf (1998) have presented an overlay of X-ray contours on an $\mathrm{H} \alpha$ 
image and $A S C A$ SIS data in two energy bands. In addition, Chu (1993) has shown a ROSAT PSPC image of the nebula.
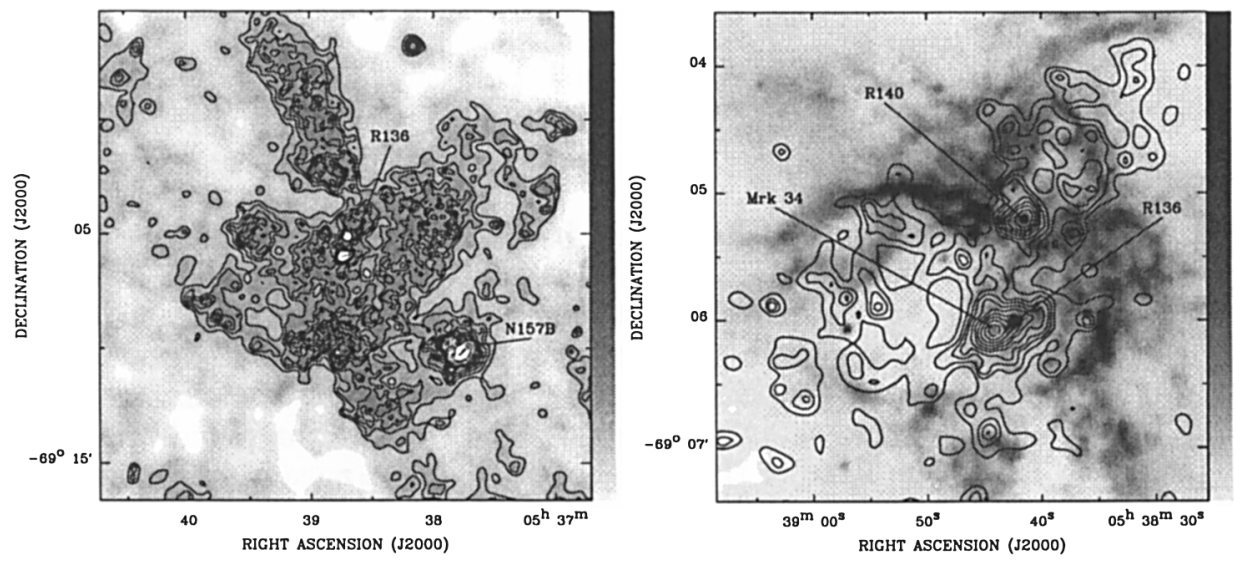

Figure 1. (left panel) X-ray image of 30 Dor. The image is a coaddition of data from ROSAT HRI exposures totaling $130 \mathrm{ks}$. The contours are at $0.6,1.2,1.9,3.0,4.5,6.4,9.1,15,24,39,63,100,160,256$, 407 , and $647 \times 10^{-3}$ counts $\mathrm{s}^{-1}$ arcmin $^{-2}$, above a local background of $\sim 1 \times 10^{-3}$. (right panel) The central region of 30 Dor in X-ray and $\mathrm{H} \alpha$. The RHRI contours start at $3 \times 10^{-3}$ counts $^{-1} \operatorname{arcmin}^{-2}$. The rest is the same as in the left panel.

These observations show that the 30 Dor nebula is an interacting complex of massive stars, dense molecular clouds, photoionized HII filaments, and diffuse hot gas. Young massive stars are concentrated in R136. But a few smaller clusters within the central region $\left(r \lesssim 4^{\prime}\right)$ may also contribute significantly to the energetics of the nebula. Dense molecular clouds typically appear in regions that are relatively dim in soft X-ray, apparently a result of displacement and/or X-ray absorption. The central region, in particular, shows hot gas bubbles surrounded by ionization fronts at boundaries of dense molecular clouds (Fig. 1).

An archival $A S C A$ observation (Fig. 2) provides $\mathrm{X}$-ray spectroscopic information about hot gas in the 30 Dor nebula (Wang 1999). The presence of individual emission lines (e.g., $\mathrm{Ne}, \mathrm{Mg}$, and $\mathrm{Si}$ ) confirms that X-ray emission in the $0.5-2 \mathrm{keV}$ band is predominantly thermal in origin. The flat spectrum at higher energies, however, suggests the presence of a nonthermal component. A power law of energy slope equal to one describes this component well, although the exact spectral shape is uncertain because of the limited energy coverage and statistics of the data. Using the Raymond \& Smith thermal plasma to characterize the thermal portion of the spectrum, I find that at least two temperature components are needed for a reasonable good fit. These two components characterize a range of hot gas temperature $0.2-1 \times 10^{7} \mathrm{~K}$ in the nebula. The high temperature component dominates in the energy band $\gtrsim 1.5 \mathrm{keV}$, and arises primarily in the core region around R136 ( $\lesssim 1^{\prime}$; Wang \& Gotthelf 1998). The 


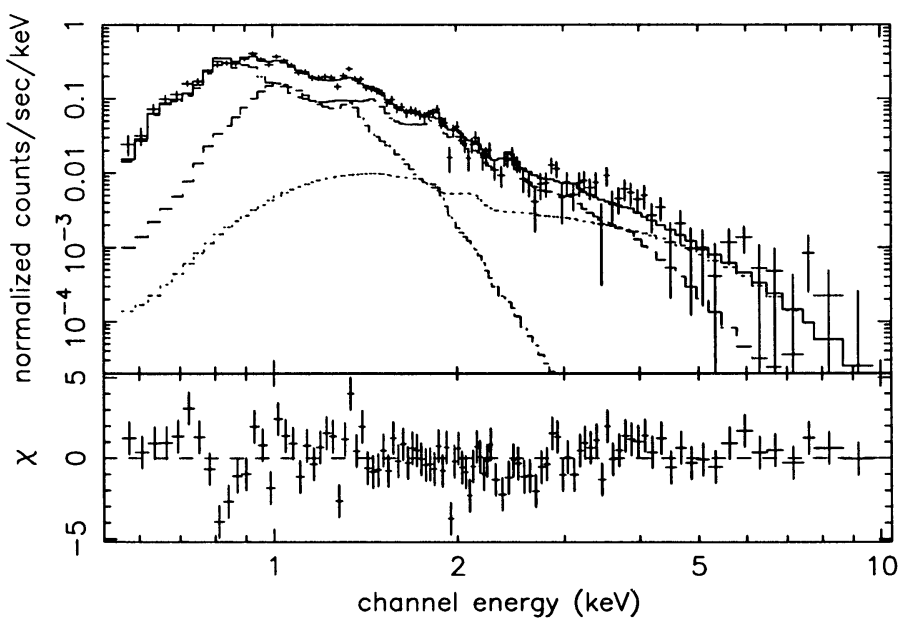

Figure 2. Average $A S C A$ SIS spectrum of the X-ray emission from 30 Dor. A three-component model fit is shown as the solid histogram. The low and high temperature thermal plasma components are plotted as dot-dashed and dashed histograms, while the power law as dotted histogram.

total inferred thermal energy and mass of hot gas are $\sim 10^{52.5} \mathrm{ergs}$ and $10^{4.6} M_{\odot}$, contained primarily in the low temperature component.

\section{Physical Processes}

The thermal energy in the hot gas is balanced by the expected mechanical energy release from stellar winds and supernovae in the nebula. The stellar winds from R136 alone have an energy release rate of $L_{w} \sim 3 \times 10^{39} \mathrm{ergs} \mathrm{s}^{-1}$ (e.g., Chu $\&$ Kennicutt 1994). Over the lifetime of R136, the integrated energy release is $\sim 10^{53} \mathrm{ergs}$, a large fraction of which should have been converted into the thermal energy of hot gas. There could also be tens of supernova explosions, although the exact number is difficult to estimate.

The thermal structure of the nebula is directly related to the cooling of hot gas. The radiative cooling rate of the hot gas is about $\sim 10^{39} \mathrm{ergs} \mathrm{s}^{-1}$, a reasonable fraction of the estimated energy input rate. The shocked stellar wind has a temperature $\sim 10^{8} \mathrm{~K}$ and is not effective in $\mathrm{X}$-ray emission. As hot gas moves from the core to the halo of the nebula, adiabatic cooling may be important. The average pressure $p / k$ is about $\sim 2 \times 10^{7} \mathrm{~K} \mathrm{~cm}^{-3}$ in the core and is a factor of $\sim 7$ lower in the halo. However, the corresponding adiabatic temperature drop of a factor $\sim 2$ is not adequate to explain the temperature difference in the two regions. The adiabatic expansion should also rarefy the gas by a factor of $\sim 3$. But the measured mean hot gas densities are comparable in the two regions. Therefore, mass loading needs to be considered.

The most effective mass loading process is likely the dynamic mixing of hot gas with HII gas. While the mass efficiency of massive star formation is 
typically only $5-10 \%$ (e.g., McKee 1989), much of the parent giant molecular cloud (GMC) of the OB association NGC 2070 is being eroded via photonevaporation. As the 30 Dor nebula has entered the so-called champagne phase (Wang 1995b), the photon-evaporated HII mass can be estimated as $\sim 0.12 M_{\odot} \mathrm{yr}^{-1}\left(S_{i} / 10^{52} \text { Ly photons s}{ }^{-1}\right)^{4 / 7}\left(t_{*} / 10^{6} \mathrm{yr}\right)^{2 / 7}\left(n_{o} / 10^{3} \mathrm{~cm}^{-3}\right)^{-1 / 7}$, where $S_{i}, t_{*}$, and $n_{o}$ are the ionizing flux of the OB association, its effective age with the present flux, and the mean density of the GMC (Whitworth 1979; Yorke et al. 1989). Only if $\sim 10 \%$ of this photon-evaporated HII gas is loaded to the shocked wind materials in the core of the nebula, can the temperature of hot gas there be explained. Over the lifetime of the nebula, the total evaporated mass is $\sim 10^{5} M_{\odot}$. In order to account for the total mass of hot gas, the mass loading over the nebula must be substantial.

The dynamic mixing is evident, right within the central cavity around R136. The cavity is clearly open to the east. The radius of the cavity, as manifested by ionization fronts, is about half an arcminute, or $\sim 7 \mathrm{pc}$. Multi-filter $H S T$ WFPC2 images (Scowen et al. 1998 and this volume) clearly demonstrate the presence of evaporative flows from the ionizing fronts into the interior of the cavity. Such flows result from the strong pressure gradient (a factor of $\sim 10$ ) between the ionization fronts and the interior of the cavity. Both the interaction between stellar winds and evaporative flows and the interface between the outflows of HII and hot gases are highly unstable. The dynamic mixing is bound to occur. Supernova blastwaves can further sporadically heat large amounts of HII gas. The high pressure in the core region tends to drive the heated gas out to the halo, leading to the blister-like morphology of the nebula.

Clearly, the study of 30 Dor has strong implications for the understanding of giant HII regions in general. The early mass loading, in particular, may determine hot gas properties of supergiant bubbles and galactic chimneys, which are all evolved from giant HII regions (Wang 1995b).

\section{References}

Chu, Y.-H. 1993, in The Soft X-ray Cosmos, AIP Conf. Proc. 313, eds: E. M. Schlegel, \& R. Petre, 154

Chu, Y.-H., \& Kennicutt Jr., R.C. 1994, ApJ, 425, 720

Chu, Y.-H., \& Mac Low, M.-M. 1990, ApJ, 365, 510

McKee, C. F. 1989, ApJ, 345, 782

Scowen, P. A., et al. 1998, ApJ, 116, 163

Wang, Q. D. 1995a, ApJ, 453, 783

Wang, Q. D. 1995b, in The Interplay between Massive Star Formation, the ISM and Galactic Evolution, eds: D. Kunth, et al., 337

Wang, Q. D., \& Helfand, D. J. 1991, ApJ, 370, 541

Wang, Q. D. 1999, ApJL, 510, L139

Wang, Q. D., \& Gotthelf, E. V. 1998, ApJ, 494, 623

Whitworth, A. 1979, MNRAS, 186, 59

Yorke, H. W., et al. 1989, A\&A, 216, 207 


\section{Discussion}

George Sonneborn: What is known about the kinematics of the hot gas?

Wang: Unfortunately, the available X-ray instruments don't yet have the spectral capability to yield any information about the kinematics. In the near future, useful information may be obtained from FUV spectroscopic measurements of elements such as OVI, which are sensitive to gas at temperatures of a few times $10^{5} \mathrm{~K}$.

Joel Bregman: Hunter told us that the R136 cluster has an age of 1-3 Myr and that the massive stars have not evolved into SN. However, your picture requires that tens of SN have gone off. Can you reconcile these apparently contradictory results?

Wang: R136 is the main central cluster of 30 Dor. But the central region contains quite a few other star clusters, which appear to be older. The presence of the X-ray binary systems does indicate $\mathrm{SN}$ in the region. There are other pieces of evidence for SN, as You-Hua has argued. Energetically, my picture does not really require any $\mathrm{SN}$, within the uncertainties of the present measurements.

Hans Zinnecker: I would like to make a comment. From your number of the hot, X-ray-emitting gas $\left(n_{e}=0.2 \mathrm{~cm}^{-3}, T=5 \times 10^{6} \mathrm{~K}\right)$, we can calculate the hot gas pressure $\left(\sim 10^{6} k_{B}\right)$. This is 10 times higher than the internal pressure of typical dense cold proto-stellar globules $\left(n=10^{4} \mathrm{~cm}^{-3}, T=10 \mathrm{~K}\right)$. So the X-ray-emitting gas can indeed trigger secondary star formation in the 30 Dor region (cf. N. Walborn review of the generation of young stars revealed by NICMOS/HST observations). 


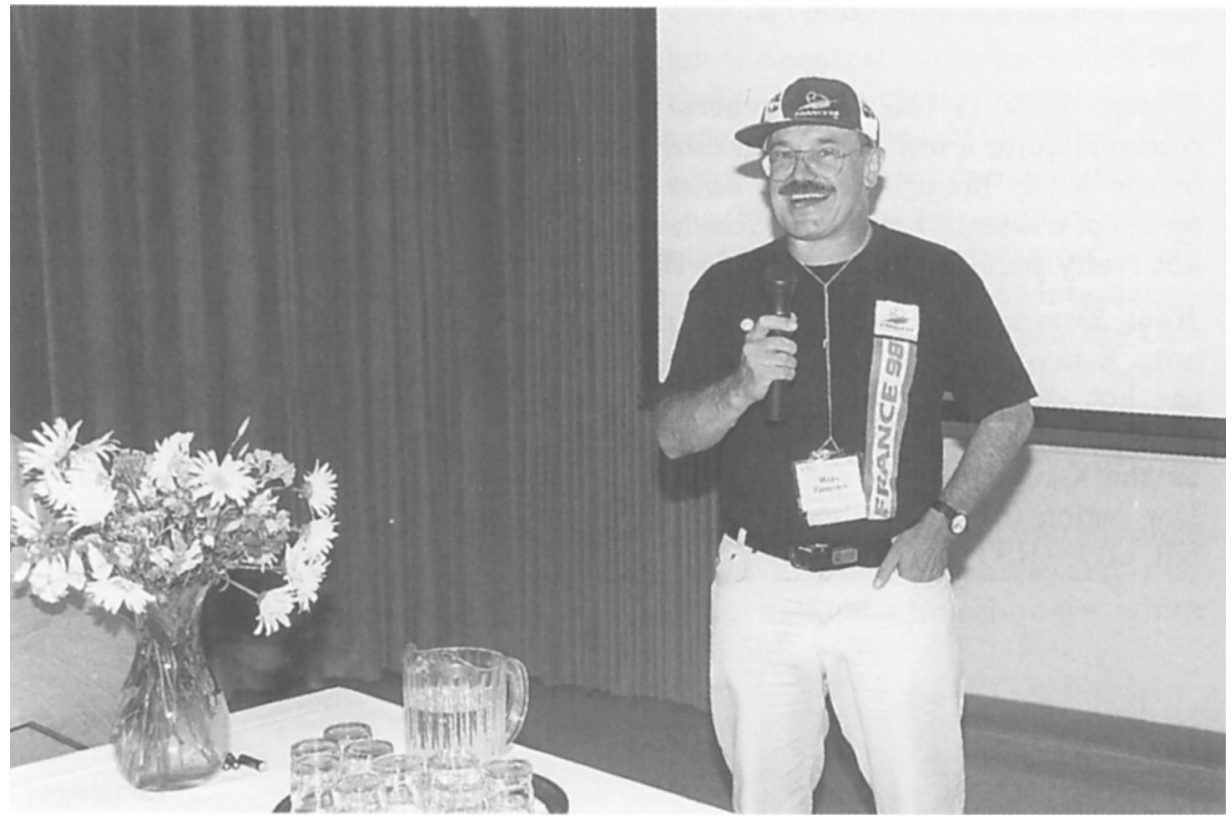

Hans Zinnecker shows his colours during his invited review. 